\title{
Primary Central Nervous System Lymphoma Presenting as Parkinsonism with Atypical MRI Findings and Elevated 14-3-3 Protein
}

\author{
Elanagan Nagarajan ${ }^{1}$ Sushma Y. Yerram² Lakshmi P. Digala ${ }^{2}$ Pradeep C. Bollu \\ ${ }^{1}$ Department of Neurology, Columbia University, New York, \\ New York, United States \\ 2Department of Neurology, University of Missouri, Columbia, \\ Address for correspondence Elanagan Nagarajan, MD,
Department of Neurology, Columbia University, New York,
NY 10032, United States (e-mail: en2452@cumc.columbia.edu).
} Missouri, United States

\begin{abstract}
Primary central nervous system lymphoma (PCNSL) is a rare neoplasm with an incidence of 2 to $3 \%$ of all CNS malignancies. The diagnosis can be challenging, especially with atypical presentations. Movement disorders can be one of the rare presentations of PCNSL. Here, we present an unusual case of gradually progressing Parkinsonism with an elevation of cerebrospinal fluid (CSF) 14-3-3 protein and atypical imaging findings found to have PCNSL. A 76-year-old female patient presented with gait and intermittent speech difficulty. Initial workup revealed a contrast-enhancing lesion in the bilateral putamen and head of caudate without any mass effect. Her symptoms were rapidly progressed over 6 months and presented with mild dysarthria, bradykinesia, mild rigidity, and reduced left arm swinging. These features were consistent with Parkinsonism. The repeat imaging showed the progression of hyperintensities in the

Keywords

- 14-3-3

- primary CNS lymphoma

- parkinsonism bilateral putamen. The patient underwent a stereotypic biopsy of the right caudate nucleus, which revealed PCNSL. She was treated with high-dose methotrexate and is currently in remission. Diagnosis of movement disorders remains clinical and rapid progression of symptoms, and atypical presentation must warrant further imaging and workup.
\end{abstract}

\section{Background}

Primary central nervous system lymphoma (PCNSL) is a rare neoplasm accounting for 2 to 3\% of CNS malignancies. Over the past few decades, there is an increasing incidence of PCNSL among immunocompetent patients, particularly in the age group of 60 to 70 years or older. It is an aggressive brain tumor with a poor prognosis. ${ }^{1}$ PCNSL is confined to the CNS and often disseminated to structures within the brain, spinal cord, ocular, and leptomeningeal layers. The clinical presentation varies, depending on the location of the tumor and the immunocompetency of the patient. ${ }^{2}$ Despite the involvement of basal ganglia in CNS lymphomas, only a handful cases have been reported with movement disorders like segmental dystonia, choreoathetosis, and Parkinson's disease. ${ }^{3-8}$
We presented an unusual case of gradually progressing Parkinsonism with an elevation of CSF 14-3-3 protein and atypical imaging findings found to have PCNSL.

\section{Case Presentation}

A 76-year-old right-handed Caucasian female patient with a history of papillary carcinoma of thyroid, hypertension, and hypothyroidism referred to our center for the evaluation of speech and walking difficulties of 6 months. She got hospitalized at a different facility for bilateral lower extremity weakness and intermittent speech problems. CT scan of the head demonstrated subtle hyperdensities in the bilateral putamen. MRI of the brain showed bilateral putamen and head of caudate T2/fluid-attenuated inversion recovery (FLAIR) 
hyperintensities that had nonhomogenous contrast enhancement without mass effect. (-Fig. 1A) Magnetic resonance angiogram and venogram did not reveal any significant vessel occlusions or cortical vein thrombosis. There was no evidence of carbon monoxide poisoning in the blood testing. With the progressive worsening of symptoms, she was referred to our clinic for an evaluation in early summer, when she was found to have mild dysarthria, bradykinesia, mild rigidity, and reduced left arm swinging. Subsequent MRIs 6 months and 8 months later ( $\mathbf{- F i g}$. 1B and C) showed the progression of the lesions with T1, T2 FLAIR hyperintensities and continued to show nonhomogenous enhancement of bilateral putamen
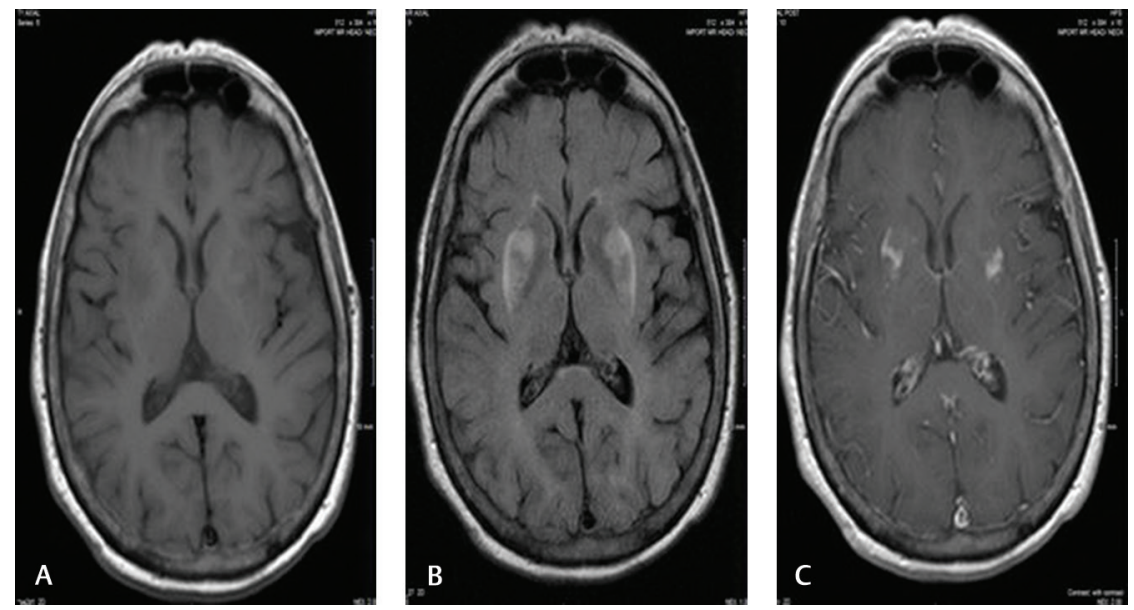

Fig. 1 First MRI of our patient 8 months before the diagnosis. (A) Isointensity to slight hypointensity in bilateral putamen in T1 sequence. (B) T2 FLAIR sequence showing bilateral putamen hyperintensities (fairly symmetric). (C) Postcontract MRI showing mild heterogenous enhancement of bilateral putamen regions. FLAIR, fluid-attenuated inversion recovery.

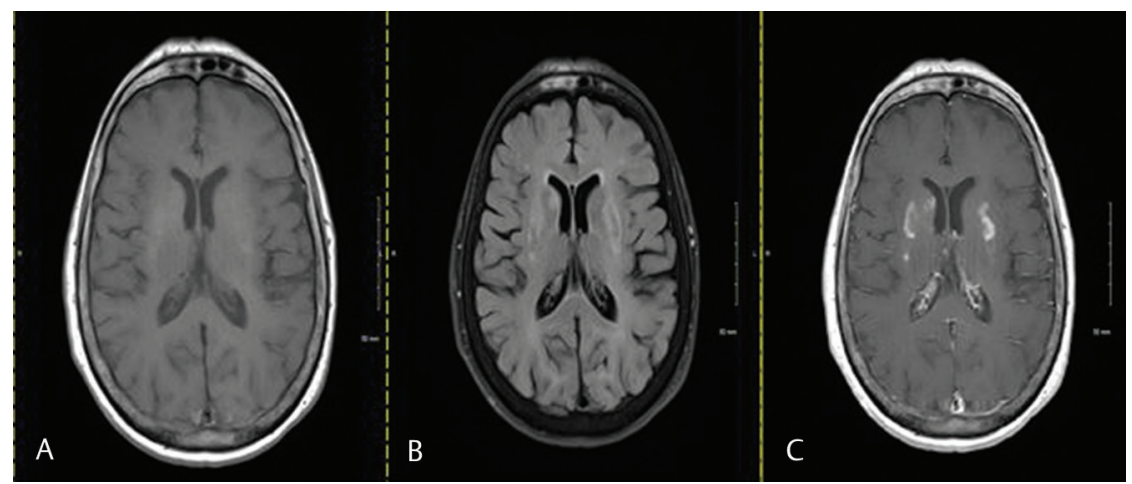

Fig. 2 MRI performed 6 months after symptoms appeared. (A) Progression of hypointensity in bilateral putamen in T1 sequence. (B) progression of T2 FLAIR sequence showing bilateral putamen hyperintensities (fairly symmetric). (C) Postcontract MRI showing progression of mild heterogenous enhancement of bilateral putamen regions. FLAIR, fluid-attenuated inversion recover.

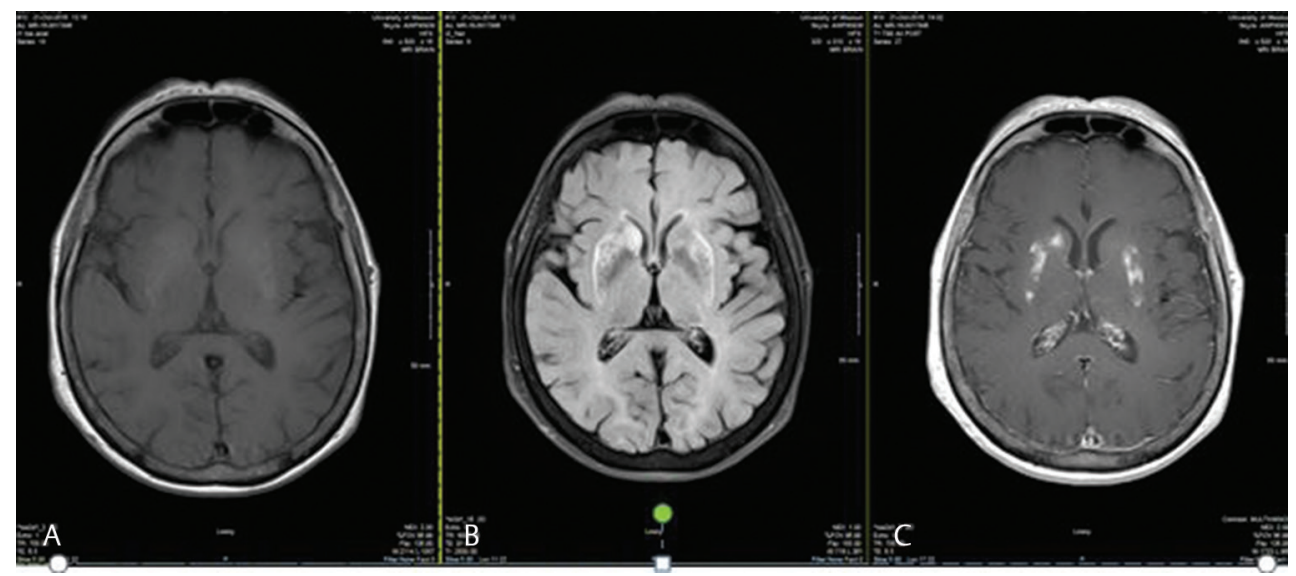

Fig. 3 (A-C) MRI shortly before tissue diagnoses confirming PCNSL. T1 and T2 FLAIR sequence of MRI showing hyper intensities in bilateral putamen and right caudate of basal ganglia. Also note similar non homogenous contrast enhancement of bilateral putamen and caudate nucleus of basal ganglia. FLAIR, fluid-attenuated inversion recovery; PCNSL, primary central nervous system lymphoma. 
regions ( $\mathbf{- F i g s . ~} 2$ and 3). Serum iron studies, liver function tests, paraneoplastic, vasculitis, hepatitis panels, toxic and heavy metal screening, HIV, and vitamin levels were found to be within normal limits/negative. Cerebrospinal fluid (CSF) analysis showed elevation of 14-3-3 protein (3.7 nanogram with cut off at 2.0), normal CSF cytology, and flow cytometry. She underwent a stereotactic biopsy of the right caudate nucleus, which showed NonHodgkin lymphoma, diffuse B cell type. The whole-body PET scan did not show extracranial lymphoma. The patient received high-dose methotrexate with remission for 4 months. She has a recurrence of lymphoma and received whole brain radiation therapy and is in remission for the past 4 months.

\section{Discussion}

Parkinsonism is a rare presentation of PCNSL, although basal ganglia involvement is common. Some of the unusual features of this case are the subacute Parkinsonian symptoms, atypical imaging features, and an elevation of 14-3-3 protein in CSF.

PCNSL commonly presents with rapidly progressive neurological deficits from solitary hemispheric masses (in immunocompetent individuals), occasionally stroke and cranial nerve deficits, and progressive encephalopathy (in immunocompromised patients ${ }^{9-11}$. Our patient presented with bradykinesia and rigidity and fulfilled the diagnostic criteria for Parkinson's disease proposed by Hughes $\mathrm{AJ}$ and et al. ${ }^{12}$ However, the red flags in our patient included abnormal MRI, which prompted further workup that included serology and CSF analysis for alternative diagnoses, which can present with Parkinsonism. Previous authors who presented cases with Parkinsonism as a symptom of basal ganglia lymphoma suggested interruption of striatal postsynaptic dopamine receptors. ${ }^{5,6}$ The unique features of our patient are that she did not have classic MRI findings of a PCNSL but later evolved to classic MRI findings with T1 and T2 FLAIR hyperintesities with homogenous contrast enhancement. ${ }^{13}$ Also, her CSF cytology and flow cytometry was negative for lymphoma, but her 14-3-3 protein was elevated with Creutzfeldt-Jakob disease in the differential diagnosis. 14-3-3 protein is found in neuronal cytoplasm, glia, and synaptic membranes of the brain. It plays a crucial role in signal transduction, neurotransmission and cell differentiation, and is released into CSF where there is extensive neuronal damage. False-positive results can be seen in patients with herpes simplex encephalitis, hypoxic brain damage, and atypical encephalitis, intracerebral metastases of bronchial carcinoma, metabolic encephalopathy, and progressive dementia of unknown cause ${ }^{14}$. In our case, it is false positive for PCNSL, which is also rare and was reported only in a few other HIV cases with PCNSL.

\section{Conclusion}

Parkinsonism is a clinical syndrome with a broad differential. The etiology of Parkinsonism is mainly clinical, based on a thorough history and clinical examination. Imaging studies are not always warranted. Our case is unique in that she had a subacute Parkinsonism with extrapyramidal signs for more than 6 months. She potentially could be diagnosed with Parkinson's disease based on queen square brain bank criteria. However, her imaging findings propelled further diagnostic testing, leading to a diagnosis of PCNSL. This case emphasizes the importance of considering imaging studies early on in selected Parkinsonian disorders to look for alternative diagnoses.

\section{Ethical Approval}

This study was approved by the Institutional Board Review of the University of Missouri Health Care-Columbia.

\section{Funding}

None.

Conflict of Interest

None declared.

\section{References}

1 Kerbauy MN, Moraes FY, Lok BH, et al. Challenges and opportunities in primary CNS lymphoma: A systematic review. Radiother Oncol 2017;122(3):352-361

2 Sierra del Rio M, Rousseau A, Soussain C, Ricard D, Hoang-Xuan K. Primary CNS lymphoma in immunocompetent patients. Oncologist 2009;14(5):526-539

3 Tan EK, Chan LL, Auchus AP, Wong MC. Reversible choreoathetosis in primary cerebral lymphoma: clinicoradiologic correlation. Eur Neurol 2003;50(1):53-54

4 Poewe WH, Kleedorfer B, Willeit J, Gerstenbrand F. Primary CNS lymphoma presenting as a choreic movement disorder followed by segmental dystonia. Mov Disord 1988;3(4):320-325

5 Pandit L, Raghotham A, Chickabasaviah Y, Khandige G, Shetty RK. Neoplastic Parkinsonism: An illustrative case report. Ann Indian Acad Neurol 2013;16(3):437-439

6 Gherardi R, Roualdes B, Fleury J, Prost C, Poirier J, Degos JD. Parkinsonian syndrome and central nervous system lymphoma involving the substantia nigra. A case report. Acta Neuropathol 1985;65(3-4):338-343

7 Cohn DF. (The Parkinsonian syndrome induced by intracranial space occupying lesions [author's transl]). Nervenarzt 1977;48(7):383-385

8 Hortelano E, Perea C, Uña E, Cebayos A, Diezhandino P, González M. Parkinsonism secondary to metastatic lesions within the central nervous system: a case report. J Med Case Reports 2010;4:218

9 Herrlinger U, Schabet M, Clemens M, et al. Clinical presentation and therapeutic outcome in 26 patients with primary CNS lymphoma. Acta Neurol Scand 1998;97(4):257-264

10 Grommes C, DeAngelis LM. Primary CNS lymphoma. J Clin Oncol 2017;35(21):2410-2418

11 Makhdoomi R, Nayil K, Rayees A, et al. Primary CNS lymphoma in immunocompetent: a review of literature and our experience from Kashmir. Turk Neurosurg 2011;21(1):39-47

12 Hughes AJ, Daniel SE, Kilford L, Lees AJ. Accuracy of clinical diagnosis of idiopathic Parkinson's disease: a clinico-pathological study of 100 cases. J Neurol Neurosurg Psychiatry 1992;55(3):181-184

13 Mansour A, Qandeel M, Abdel-Razeq H, Abu Ali HA. MR imaging features of intracranial primary CNS lymphoma in immune competent patients. Cancer Imaging 2014;14:22

14 Satoh J, Kurohara K, Yukitake M, Kuroda Y. The 14-3-3 protein detectable in the cerebrospinal fluid of patients with prionunrelated neurological diseases is expressed constitutively in neurons and glial cells in culture. Eur Neurol 1999;41(4): 216-225 